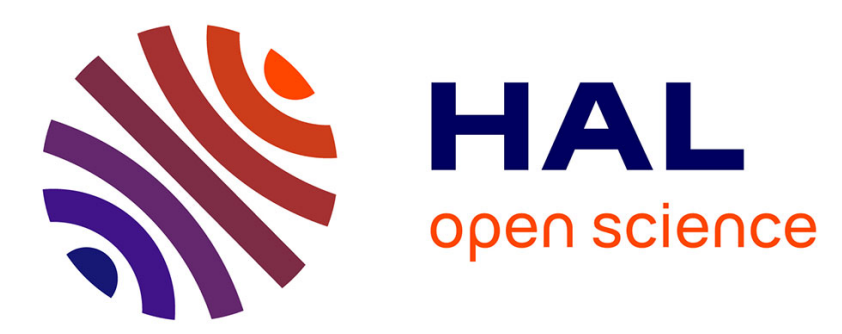

\title{
Additional Sound Absorption Within a Poroelastic Lamella Network Under Oblique Incidence
}

Nicolas Dauchez, Benoit Nennig, Olivier Robin

\section{To cite this version:}

Nicolas Dauchez, Benoit Nennig, Olivier Robin. Additional Sound Absorption Within a Poroelastic Lamella Network Under Oblique Incidence. Acta Acustica united with Acustica, 2018, 104 (2), pp.211219. 10.3813/AAA.919162. hal-01726398v2

\section{HAL Id: hal-01726398 \\ https://hal.science/hal-01726398v2}

Submitted on 7 Feb 2019

HAL is a multi-disciplinary open access archive for the deposit and dissemination of scientific research documents, whether they are published or not. The documents may come from teaching and research institutions in France or abroad, or from public or private research centers.
L'archive ouverte pluridisciplinaire HAL, est destinée au dépôt et à la diffusion de documents scientifiques de niveau recherche, publiés ou non, émanant des établissements d'enseignement et de recherche français ou étrangers, des laboratoires publics ou privés. 


\title{
Additional sound absorption within a poroelastic lamella network under oblique incidence
}

\author{
Nicolas Dauchez ${ }^{1}$, Benoit Nennig ${ }^{2}$, and Olivier Robin ${ }^{3}$ \\ ${ }^{1}$ Sorbonne Universités, Université de Technologie de Compiègne, Laboratoire Roberval, \\ UMR CNRS 7337, CS 60319, 60203 Compiègne cedex, France. \\ ${ }^{2}$ Institut supérieur de mécanique de Paris (SUPMECA), Laboratoire Quartz EA 7393, \\ 3 rue Fernand Hainaut, 93407 Saint-Ouen, France. \\ ${ }^{3}$ Groupe d'Acoustique de l'Université de Sherbrooke (GAUS), \\ Université de Sherbrooke, 2500, Bd de l'Université, Sherbrooke J1K2R1 Canada.
}

\begin{abstract}
This archived file is not the final published version of "N. Dauchez, B. Nennig, and O. Robin. Additional sound absorption within a poroelastic lamella network under oblique incidence. Acta Acust. United Ac., 104(2):211-219, 2018." (C) (2018) S. Hirzel Verlag/European Acoustics Association. Contact the publisher for reprint or permission to use the material in any form. The definitive publisher-authenticated version is available online at http://dx.doi.org/10.3813/AAA.919162.
\end{abstract}

\section{Abstract}

To tackle the lack of efficiency of passive sound absorbing treatments in the low frequency range, a specific arrangement of a poroelastic material is proposed. Compared with the usual uniform layout, giving a lamella network structure to a material provides additional sound absorption below the quarter wavelength resonance frequency at oblique incidence. This is demonstrated experimentally on a sample made of melamine foam lamellas. A numerical approach that incorporates geometric periodicity highlights the mechanisms involved in this additional dissipation, achieved by combining structural and viscous dissipation within the lamellas. The shear and bending resonances of the lamellas can be excited at oblique incidence. The frequency of the shear resonance is related to the thickness of the sample, whereas the bending resonance is also a function of the width of the lamellas. These simple dimensional parameters allow adjustable tuning of the associated frequencies at which additional sound absorption can be obtained.

\section{Introduction}

Passive sound absorbing materials like foam, wool and microperforated panels are generally efficient at frequencies for which the acoustic wavelength is in the order of magnitude of the material's thickness. There is still a need for compact, light but also effective sound absorbing materials, though their configuration presents a challenge, especially at low frequencies. This topic has received considerable attention and metamaterial and metasurface approaches have recently given new impetus to the design of such materials $[1,2]$.

Indeed, in addition to the viscothermal losses present in porous materials, several other mechanisms have been used to increase dissipation efficiency. For instance, double porosity materials that use the pressure diffusion effect between the micro and the macro pore networks have been proposed $[3,4,5,6]$. Another approach is to excite trapped modes between a rigid inclusion and a rigid wall $[7,8]$.

In order to enhance low frequency sound absorption, quarter wavelength $[9,10]$ (eventually coiled [11]), split ring and Helmholtz resonators [12, 13, 14, 15, 6] have been embedded in porous materials. Such resonators can dramatically boost sound absorption provided that the resonator frequency is above the Biot frequency (i.e., transition from a viscous to an inertial regime). They can also be combined to create a slow sound channel $[16,10,17,18]$.

Instead of fluid resonators, membranes [19], thin plates [20] and shell resonances $[21,6]$ have also been considered. These elastic resonators usually resonate at lower frequencies than their fluid counterparts, and avoid the boundary layer thickness constraint that can typically limit the Helmholtz resonator neck diameter.

Recent works have provided interesting ways of tuning the resonators to theoretically obtain total sound absorption at prescribed frequencies, based on the critical coupling phenomenon $[20]$, i.e., by balancing the energy leakage and the internal losses in the resonator. Following this approach, nearly perfect sound absorption was obtained for a material thickness of 1/88-wavelength [18], while considerable sound absorption was obtained with a viscoelastic plate [20].

The aim of this paper is to design multifunctional, light and compact noise reducing treatments based on poroelastic materials, like most of the previously cited studies, but the latter mainly focused on an equivalent fluid model and ignored skeleton elasticity. Due to the coupling between the fluid phase and the solid phase, the wealth of physical properties of poroelastic materials can 


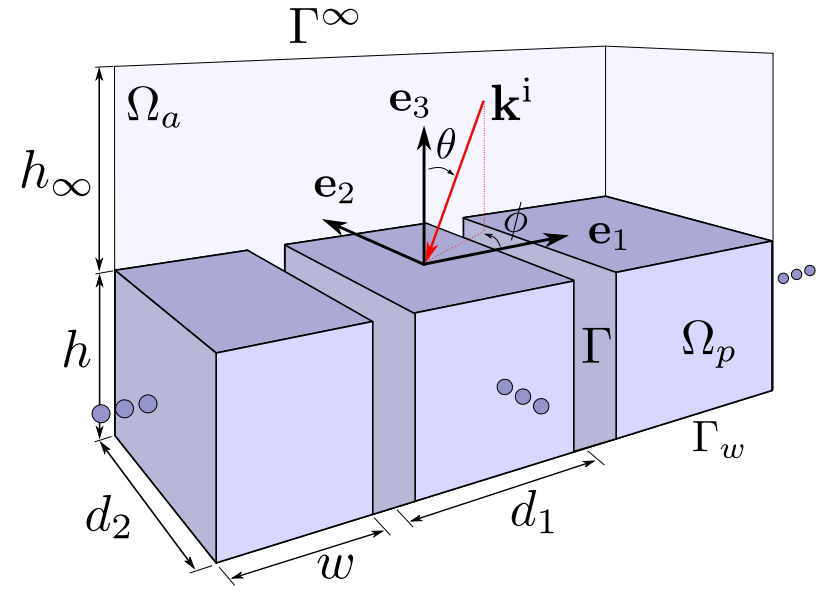

Figure 1: Geometry of the problem.

be used to couple fluid and elastic solid resonances in a single material and thus enhance sound absorption for various applications. To illustrate the different effects of the skeleton, a simple configuration made of a periodic arrangement of porolelastic lamellas and supported by a rigid backing as in Ref. [22] was chosen. When coupled with an acoustic excitation at oblique incidence, additional absorption peaks were observed linked to the bending resonance or shear resonance of the frame [23]. The lamella dimensions were chosen so that the absorption coefficient is boosted in the low frequency range by the shear and bending resonances.

The present paper is organized as follows. After presenting the material geometry and its numerical model using the finite element method (FEM) in Sec. 2, a description of the experimental protocol chosen to perform the oblique incidence measurements is described. Finally, numerical and experimental results are presented in Sec. 4 to illustrate the contribution of shear and bending resonances to the absorption coefficient.

\section{Problem statement}

Let us consider the time-harmonic (with the time convention $\mathrm{e}^{-\mathrm{i} \omega t}$ ) scattering problem depicted in Figure 1. The configuration consists of a periodic array of poroelastic strips. The foam $\Omega_{p}$ is clamped on a rigid wall and embedded in an unbounded fluid domain $\Omega_{a}$ on the top and on the lateral faces. In direction $\mathbf{e}_{1}$, the configuration has a period $d_{1}$, whereas in direction $\mathbf{e}_{2}$ the system is assumed infinite and modeled by an arbitrary period.

The acoustic wave propagation in the poroelastic domain $\Omega_{p}$ is governed by the Biot equation [24, Chap. 6]. The foam strips are clamped on $\Gamma_{w}$ and surrounded by lateral air gaps. Classical coupling conditions apply at the air-foam interface $\Gamma$ [24, Chap. 13].

The total pressure must satisfy the Helmholtz equation in the surrounding fluid domain $\Omega_{a}$ and in the air gaps

$$
\Delta p+k_{a}^{2} p=0
$$

where $k_{a}$, denotes the air wavenumber. The fluid has a density $\rho_{a}$ and a sound speed $c_{a}$.

The incident pressure field is a plane wave $p^{\mathrm{i}}=A^{\mathrm{i}} \mathrm{e}^{\mathrm{i} \mathbf{k}^{\mathrm{i}} \cdot \mathbf{x}}$ impinging on the material with the wavenumber $\mathbf{k}^{\mathrm{i}}=$ $-k_{a}(\sin \theta \cdot \cos \phi, \sin \theta \cdot \sin \phi, \cos \theta)$. Its direction is defined by the inclination $\theta$ and azimuth $\phi$ of the spherical coordinate system. Due to the periodicity of the geometry, both the incident and the scattered fields are pseudoperiodic (i.e. d-periodic with a phase shift). Indeed, each physical variable (called $X$ ) satisfies the relation

$$
X(\mathbf{x}+\mathbf{d}, \omega)=X(\mathbf{x}, \omega) \mathrm{e}^{\mathrm{i} \mathbf{k}_{\perp}^{\mathrm{i}} \cdot \mathbf{d}},
$$

with $\mathbf{d}=\left(d_{1}, d_{2}, 0\right)$ and the in-plane component of the incident wavenumber $\mathbf{k}_{\perp}^{\mathrm{i}}$. This property allows substantial simplifications because only one elementary cell has to be meshed.

The radiation condition of the scattered field in the upper air domain is implemented with a 'Dirichlet to Newman' map based on the Floquet decomposition on the plane boundary $\Gamma_{\infty}$ at height $h_{\infty}$. Here, the total pressure reads $p=p^{\mathrm{i}}+p^{\mathrm{r}}$ and the reflected pressure can be expanded as

$$
\left.p^{\mathrm{r}}(\mathbf{x}, \omega)\right|_{\Gamma_{\infty}}=\sum_{m, n \in \mathbb{Z}^{2}} A_{m n} \phi_{m, n}(\mathbf{x}) \mathrm{e}^{\mathrm{i} k_{3 m n} h_{\infty}},
$$

with

$$
\phi_{m, n}(\mathbf{x})=\frac{1}{\sqrt{S}} \mathrm{e}^{\mathrm{i}\left(k_{1 m} x_{1}+k_{2 n} x_{2}\right)}
$$

where $A_{m n}$ are the amplitudes of the Floquet mode $(n, m), k_{1 m}=k_{1}^{\mathrm{i}}+m \frac{2 \pi}{d_{1}}, k_{2 n}=k_{2}^{\mathrm{i}}+n \frac{2 \pi}{d_{2}}, k_{3 m n}=$ $\sqrt{k_{a}^{2}-k_{1 m}^{2}-k_{2 n}^{2}}$, and $S=d_{1} \times d_{2}$ is the surface of the elementary cell. To satisfy the radiation condition, i.e. the field remains bounded when $x_{3} \rightarrow \infty$, the values of $k_{3 m n}$ are chosen to consider both propagative and evanescent waves in $\Omega_{a}$. The highest Floquet mode index taken into account in each direction is chosen as the number of cuton Floquet modes +2 . This approach was preferred here as the use of the Perfect Matching Layer (PML) [25] technique is not efficient for "low-frequency" applications, i.e. when the wavelength is large compared to the size of the computational domain.

The integration of the acoustic intensity relation over the unit cell using the orthogonality of the Floquet modes leads to the power balance. The power reflection coefficient $\mathcal{R}$ is defined as the ratio of the scattered power in the $\mathbf{e}_{3}$ direction

$$
\mathcal{P}_{r}=\sum_{m, n \in \mathbb{Z}^{2}} \operatorname{Re}\left(k_{3 m n}\right)\left|A_{m n}\right|^{2} /\left(\rho_{a} \omega\right),
$$

to the incident power

$$
\mathcal{P}_{\mathrm{i}}=S\left|A^{\mathrm{i}}\right|^{2} k_{3}^{\mathrm{i}} /\left(\rho_{a} \omega\right) .
$$

The absorption coefficient $\alpha$ is then defined as the ratio of the absorbed power to the incident power

$$
\alpha=1-\mathcal{R} \text {. }
$$


The problem is solved by FEM using Lagrange quadratic tetrahedral finite elements. The $(\mathbf{u}, p)$ formulation [26] is used In the poroelastic domain $\Omega_{p}$. Coincident meshes on each opposite lateral boundary of the periodic cell are used [27] to facilitate the use of the periodicity condition recalled in equation (2). Unstructured meshes are employed in the remainder of the computational domain. This results in a model with approximatively 25000 degrees of freedom.

\section{Experimental methods}

Measurements at oblique incidence are necessary to illustrate the contribution of the shear and bending resonances of the porous lamella network. This section describes the experimental protocol chosen and the properties of the sample tested.

\subsection{Methods for measuring sound ab- sorption at oblique incidence}

Measurements of the properties of sound absorbing materials at oblique incidence are not trivial and have been studied extensively. The impedance tube method is mainly intended for measurements under an acoustic plane wave at normal incidence. Improvements of this method so that it can measure absorption coefficients and impedance at oblique incidence were proposed, including an apparatus for prescribed incidence angles that had been suggested many years ago [28] and the more recent multi-modal decomposition method [29]. An important limitation of the impedance tube method is that the dimensions of the sample are generally small, and even if the minimum size of samples in the impedance tube have been shown to be slightly expanded [30], out-of-tube methods are generally preferred since they generally allow simpler setups and testing large samples.

Temporal separation and acoustic field approaches can mainly be distinguished (see a review paper in [31]), the latter mostly having been applied following the transfer function method using two microphones, a sound source and different field assumptions (plane or spherical waves, with fixed [32] or rotating samples [22] in anechoic rooms). The first alternative approach of which mention can be made is the increasingly common use of spherical microphone arrays [33]. Another interesting method is that used by Tamura [34] who proposed a measurement procedure using the spatial Fourier transform for the decomposition of sound pressures measured in two planes above a sample into the incident and reflected sound pressures (with a fixed sound source). This method is generally considered complex and very time consuming [31].

In the present work, the approach taken uses two fixed microphones and a mobile source [35]. It was first applied to the measurement of the sound absorption coefficient of a material under a synthesized diffuse acoustic field excitation at the material surface, by using a synthetic array of acoustic monopoles facing the material. Here, it is extended to measurements under acoustic plane waves with prescribed incidence angles.

\subsection{Description of the method applied}

The classical two-microphone approach and a sourceimage model [32] is used to build a reflection coefficient database by translating a point source over a plane parallel to the material surface (see Figure 2). The sound absorption coefficient under a synthetic pressure field is then calculated during a post-processing phase.

Under the assumption of an ideal point source placed at a given position $\mathbf{x}_{i}(i=1, I)$ at a height $z=z_{3}$ above a layer of porous material, the acoustic pressure field at microphone $M_{j}$ at height $z=z_{j}(j=1,2)$ can be written (spherical decoupling hypothesis) as:

$$
\tilde{p}_{i j}\left(\mathbf{x}_{i}, \omega\right)=\rho_{a} q_{i}(\omega)\left(\frac{\mathrm{e}^{\mathrm{i} k_{a} r_{i j}}}{r_{i j}}+R\left(\mathbf{x}_{i}, \omega\right) \frac{\mathrm{e}^{\mathrm{i} k_{a} r_{i j}^{\prime}}}{r_{i j}^{\prime}}\right),
$$

where $q_{i}(\omega)$ is the source volume acceleration, $r_{i j}$ and $r_{i j}^{\prime}$ are the distances between the microphone $M_{j}$ and the source or the image source at the $i$-th position, and finally $R\left(\mathbf{x}_{i}, \omega\right)$ is the reflection coefficient of the material surface corresponding to the $i$-th position of the point source. The measurement of $H\left(\mathbf{x}_{i}, \omega\right)=\tilde{p}_{i 2}\left(\mathbf{x}_{i}, \omega\right) / \tilde{p}_{i 1}\left(\mathbf{x}_{i}, \omega\right)$ allows calculating the pressure reflection coefficient for a given incidence angle using the classical relation [32]

$$
R\left(\mathbf{x}_{i}, \omega\right)=\frac{\frac{\mathrm{e}^{\mathrm{i} k_{a} r_{i 2}}}{r_{i 2}}-H\left(\mathbf{x}_{i}, \omega\right) \frac{\mathrm{e}^{\mathrm{i} k_{a} r_{i 1}}}{r_{i 1}}}{H\left(\mathbf{x}_{i}, \omega\right) \frac{\mathrm{e}^{\mathrm{i} k_{a} r_{i 1}^{\prime}}}{r_{i 1}^{\prime}}-\frac{\mathrm{e}^{\mathrm{i} k_{a} r_{i 2}^{\prime}}}{r_{i 2}^{\prime}}}
$$

In practice, a square sample of porous material of sidelength $L$ and thickness $h$ is placed on a rigid impervious backing. The synthetic source array and the two microphones $M_{1}$ and $M_{2}$ are centered on the material's surface. Using the two-microphone method described previously, the reflection coefficient can be measured under various incidence angles corresponding to successive source positions $i$ of point sources, thus creating a virtual array of monopoles in front of the material surface.

It can be shown (details can be found in [35]) that with a database of measured reflection coefficients $R\left(\mathbf{x}_{i}, \omega\right)$ and a calculated cross spectral density (CSD) matrix of source volume accelerations $\mathbf{S}_{Q Q}$, the power reflection coefficient $\mathcal{R}_{s}(\omega)$ under a synthesized pressure field during a post-processing phase is obtained according to: Eq. (10) below

$$
\mathcal{R}_{s}=\frac{\mathbf{h}_{1}{ }^{H} \mathbf{S}_{Q Q} \mathbf{h}_{1}}{\mathbf{g}_{1}^{\prime H} \mathbf{S}_{Q Q} \mathbf{g}_{1}^{\prime}}
$$

where $\mathbf{g}_{1}^{\prime}=\left[g_{11}^{\prime}, \ldots, g_{i 1}^{\prime}, \ldots, g_{I 1}^{\prime}\right]^{T}, \quad \mathbf{h}_{1}=$ $\left[R\left(\mathbf{x}_{1}, \omega\right) g_{11}^{\prime}, \ldots, R\left(\mathbf{x}_{i}, \omega\right) g_{i 1}^{\prime}, \ldots, R\left(\mathbf{x}_{I}, \omega\right) g_{I 1}^{\prime}\right]^{H} \quad$ with the source positions ranging in $(i=1, I)$ and ${ }^{T}$ and $H$ denote the transpose and Hermitian transpose, respectively, the Green's functions corresponding to 

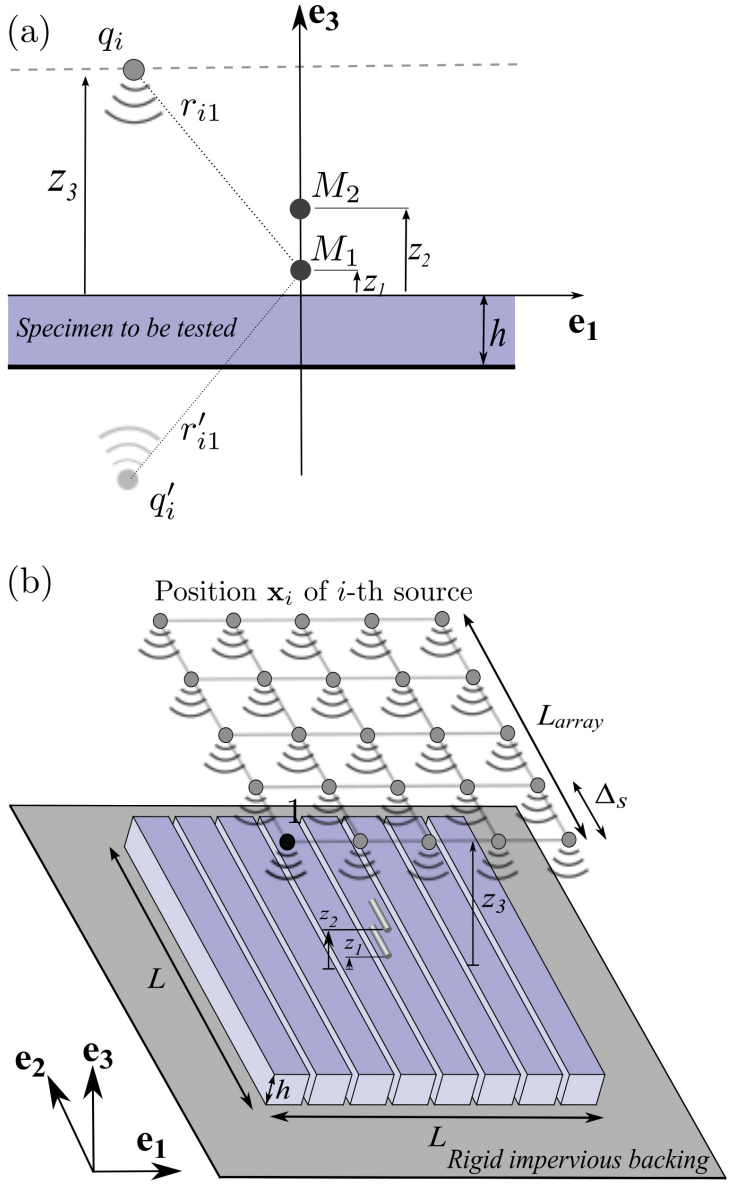

Figure 2: (a) Description of the problem and coordinate system for a spherical wave model using a single point source - (b) Description of the problem using a $i$-source array (the side-length of the virtual array is $L_{\text {array }}$, with uniformly distributed sources positioned with the same source separation $\Delta_{s}$ ) [Adapted from [35]].

the propagation from the real (image) point source to the microphone $M_{j}$ are denoted $g_{i j}(\omega)=\frac{\mathrm{e}^{\mathrm{i} k_{a} r_{i j}}}{r_{i j}}$ $\left(g_{i j}^{\prime}(\omega)=\frac{\mathrm{e}^{\mathrm{i} k_{a} r_{i j}^{\prime}}}{r_{i j}^{\prime}}\right.$, respectively).

Note that the CSD matrix of the source volume acceleration $\mathbf{S}_{Q Q}$ can be calculated using a Wave Field Synthesis [36], a Planar Nearfield Acoustical Holography [37] or a Least-Squares [38] approach. The target pressure field is defined here by the CSD of a unitary propagating plane wave.

$$
\tilde{p}(\mathbf{x}, \omega)^{\mathrm{i}}=\exp \left(\mathrm{ik}^{\mathrm{i}} \cdot \mathbf{x}\right)
$$

as defined in sec. 2 .

The corresponding absorption coefficient for the synthetized incident pressure field is $\alpha_{s}(\omega)=1-\mathcal{R}_{s}$, and is a function of $\theta$ and $\phi$ as defined in Figure 1.

\subsection{Measurement setup}

A melamine foam of thickness $h=25.4 \mathrm{~mm}$ (1 inch) was used to prepare the specimen of approximately
$1.2 \times 1.2 \mathrm{~m}^{2}$ area, mounted on a rigid laminated chipboard. Lamellas were cut out from a large sample using a band saw with a razor-edge band knife. The network was composed of 65 lamellas of $w=15 \mathrm{~mm}$ width glued one after the other on a rigid laminated chipboard, each being separated by a distance of $d_{1}-w=3.6 \mathrm{~mm}$. Care was taken to ensure regular gluing at the bottom of the lamellas (spray adhesive) and regular spacing. Compared with a homogeneous layer of a similar area, the lamella network is finally composed of nearly $20 \%$ less sound absorbing material (see Figure 3(c)). It is noteworthy that due to the period $d_{1}=18.6 \mathrm{~mm}$, only the fundamental Floquet mode $k_{300}$ propagates up to $18 \mathrm{kHz}$. In the frequency band measured the lamella specimen behaves like a homogenized material where only specular reflection is present.

The specimen tested was laid directly on the floor of the hemi-anechoic room (see Figures 3(a-b)). A small loudspeaker (Gallo Nucleus 3 in.) was translated manually using a rigid aluminum frame on a mesh with $7 \times 7$ positions at a height $z_{3}=0.27 \mathrm{~m}$ above the material surface, the center source position corresponding to the normal incidence case. Each source position was separated by $\Delta_{s}=0.15 \mathrm{~m}$ in both $x$ and $y$ directions, leading to an array side-length of $L_{\text {array }}=0.9 \mathrm{~m}$. Two microphones (PCB 1/4 in.) were positioned at the center of the samples at heights $z_{1}=6 \mathrm{~mm}$ and $z_{2}=56 \mathrm{~mm}$, respectively, and were calibrated for amplitude. The maximum incidence angle $\theta_{\max }$ that can be included in the database of the measured reflection coefficients $R\left(\mathbf{x}_{i}, \omega\right)$ was defined by the source to reproduction plane separation $z_{s}$ and the longest source to microphone distance, in this case $\theta_{\max } \approx 67^{\circ}$. For each source position, a logarithmic swept sine $(200 \mathrm{~Hz}$ to $2000 \mathrm{~Hz}$, in one second) was used to drive the loudspeaker, and the transfer function $H$ between the two microphones was estimated using 10 consecutive averages.

The properties of the foam are provided in Table 1. The porosity is measured by using the pressure/mass method. The resistivity is determined according to the ASTM C522 standard. The tortuosity is measured by an ultrasonic method. The two characteristic lengths are obtained by analytical inversion based on the 3 microphone in-duct technique.

To ensure the reliability of the viscoelastic properties, they were determined from the bending vibratory response of several lamellas within the network. Contactless vibration measurements were performed using a scanning laser vibrometer. Small patches of reflective material were applied to the top of one lamella in order to capture the vibration response to an impact (see Figure 4). A soft impactor (laboratory tip applicator) was used to excite the bending motion at several points, and the vibratory measurement was triggered on the vibrometer signal. Two examples of typically acquired signals are provided in Figure 4, clearly showing the impulse response of a single damped mode after the shock. From these signals, the loss factor and the bending resonance 
Table 1: Measured material parameters.

\begin{tabular}{|c|c|c|c|c|c|c|c|c|}
\hline $\begin{array}{c}\text { Tortuosity } \\
\alpha_{\infty} \\
{[-]}\end{array}$ & $\begin{array}{c}\text { Porosity } \\
\Phi \\
{[-]}\end{array}$ & $\begin{array}{c}\text { Resistivity } \\
\sigma \\
{\left[\mathrm{Nm}^{-4} \mathrm{~s}\right]}\end{array}$ & $\begin{array}{c}\text { Viscous } \\
\text { length } \Lambda \\
{[\mu \mathrm{m}]}\end{array}$ & $\begin{array}{c}\text { Thermal } \\
\text { length } \Lambda^{\prime} \\
{[\mu \mathrm{m}]}\end{array}$ & $\begin{array}{c}\text { Foam mass } \\
\text { density } \rho_{1} \\
{\left[\mathrm{~kg} \cdot \mathrm{m}^{-3}\right]}\end{array}$ & $\begin{array}{c}\text { Young's } \\
\text { modulus } E \\
{[\mathrm{kPa}]}\end{array}$ & $\begin{array}{c}\text { Poisson } \\
\text { ratio } \nu \\
{[-]}\end{array}$ & $\begin{array}{c}\text { Loss } \\
\text { factor } \eta \\
{[-]}\end{array}$ \\
\hline 1 & 0.98 & 7920 & 132 & 149 & 6.1 & 120 & 0 & 0.075 \\
\hline
\end{tabular}

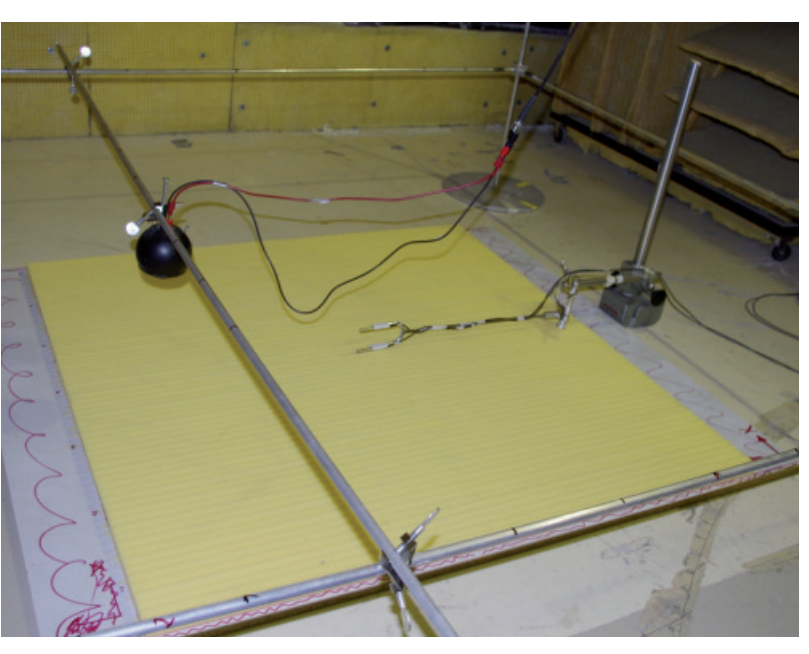

(a)

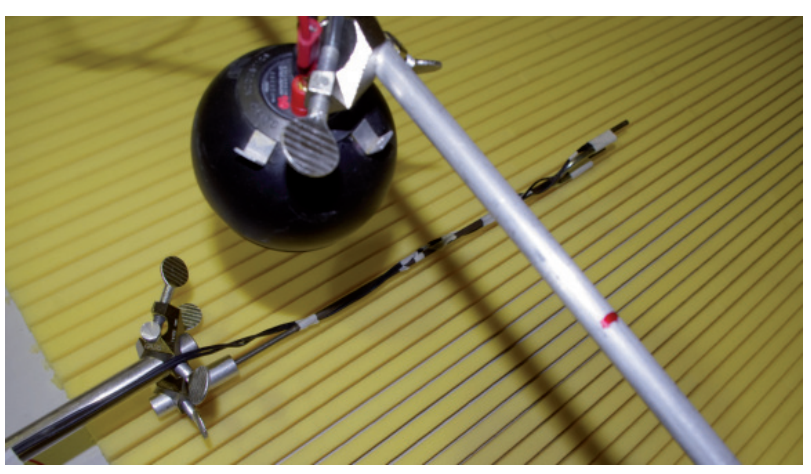

(b)

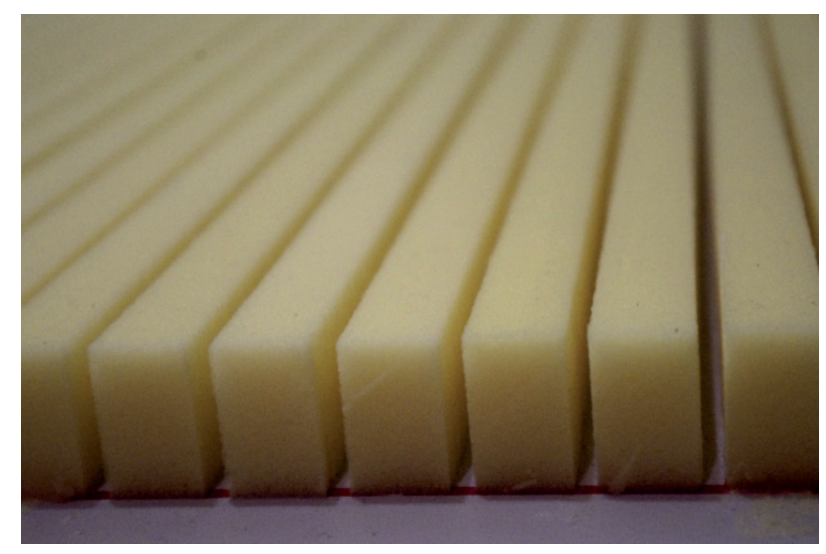

(c)

Figure 3: Experimental set-up for the case of the lamella network (a); Zoom on the source and microphones (b); Zoom on the poroelastic lamella network (c). frequency were both estimated, with mean values of 0.075 and $500 \mathrm{~Hz}$, respectively. A null value was assumed for this parameter since numerical simulation showed that the Poisson ratio had no significant effect on the resonance frequencies of the lamellas. Under this hypothesis, the Young's modulus can be easily determined.

Here the dimensions of the lamellas were chosen to ensure a bending resonance frequency above $400 \mathrm{~Hz}$ because of experimental limitations (see Sec. 4.2). Changing the aspect ratio made it possible to shift this frequency up or down for practical applications.

\section{Results and discussion}

\subsection{Numerical results}

The numerical results were computed with the material properties given in Table 1, while the dimensions are given in section 3.3 for several incidence angles. As seen in Figure 1, $\theta$ is the angle defined with respect to the vertical axis $\mathbf{e}_{3}: \theta=0^{\circ}$ states for a normal incidence. This is usually referred to as the inclination angle. $\theta=90^{\circ}$ states for a grazing incidence. In the experimental configuration, the maximum angle available is $67^{\circ}$. The azimuthal angle $\phi$ defined with respect to the horizontal axis $\mathbf{e}_{1}$ is related to the orientation of the lamellas. Here, $\phi=0^{\circ}$ states for an incidence normal to the lamellas axis oriented by $\mathbf{e}_{2}$, whereas $\phi=90^{\circ}$ states for an incidence parallel to the axis of the lamellas.

Figure 5 shows the numerical results for several incidence angles on the lamella network. When $\theta=0^{\circ}$, the angle $\phi$ has no effect: the bottom curve in figures $5(\mathrm{a}-\mathrm{c})$ is always the same. A dip can be noticed around a frequency of $1400 \mathrm{~Hz}$, due to the quarter wavelength mode related to the longitudinal solid borne wave whose frequency can be approximated by $f_{l}=\frac{1}{2 \pi} \frac{\sqrt{E / \rho_{1}}}{4 h}$, which gives $1380 \mathrm{~Hz}$.

A comparison with the double porosity (DP) model for slits [4] which assumes no motion of the skeleton is presented in Figure 5(a) and provides another validation of the numerical model. It can be seen that the DP and FEM models are the same below the frequency $f_{l}$. The elasticity of the skeleton seems to lower the absorption coefficient above this frequency. In comparison with the homogeneous layer at normal incidence (see Figure 6), the sound absorption coefficient is slightly lower for the lamella network since the fraction of porous material is smaller. It is noteworthy that this can be avoided by tuning the resistivity, as in $[5,39]$ to obtain the DP pressure 

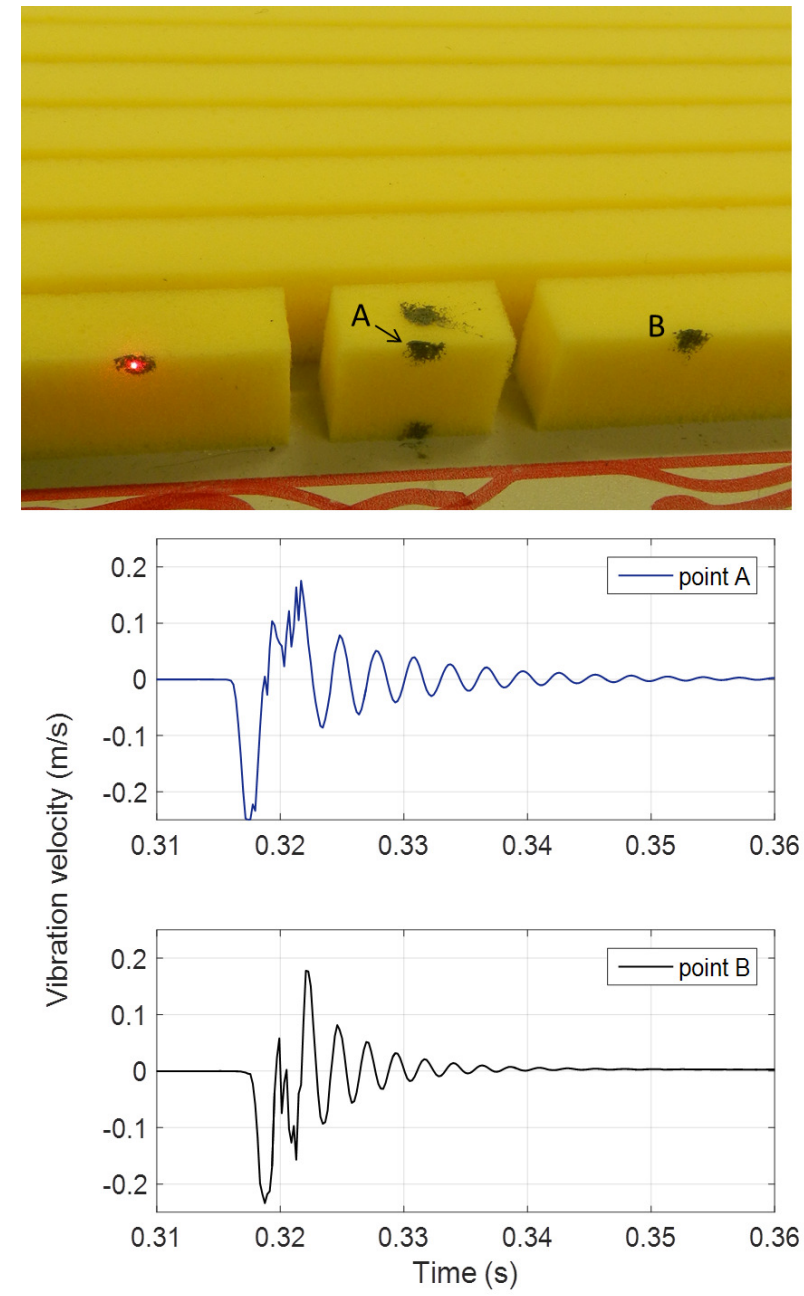

Figure 4: Experimental measurement of the vibratory response to an impact of a lamella: (top) tested sample, (bottom) free response versus time at two locations (A: beam-like sample; B: continuous lamella).

\section{diffusion effect[4].}

Figure 5(a) shows the effect of the inclination angle $\theta$ for an azimuthal angle $\phi$ of 0 degrees. It shows that a peak appears just before $500 \mathrm{~Hz}$ as long as $\theta$ increases. In these conditions (incidence normal to the lamella axis), the bending mode of the lamellas is considerably excited. Its frequency can be approximated by considering the first bending mode of the uniform clamped-free beam [40] by

$$
f_{b}=\frac{1.875^{2}}{2 \pi h^{2}} w \sqrt{\frac{E}{12 \rho_{1}}},
$$

which gives $526 \mathrm{~Hz}$. This frequency is slightly overestimated since shear effects are not accounted for in this approximation. This difference becomes smaller when the ratio $h / w$ increases.

Figure 5(b) shows that when $\phi=45^{\circ}$, another peak appears around $1000 \mathrm{~Hz}$, whereas the first peak is less pronounced. This second peak is related to the shear mode of the lamellas whose frequency can be approxi-

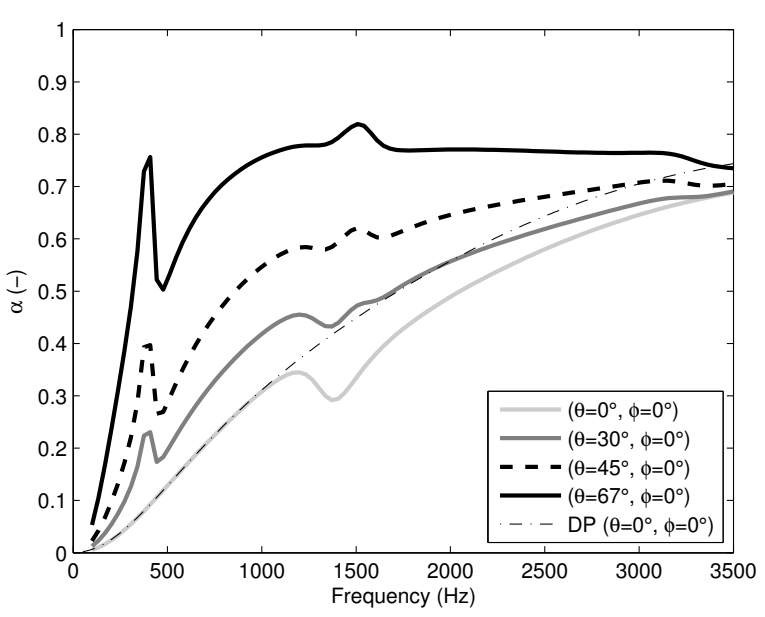

(a)

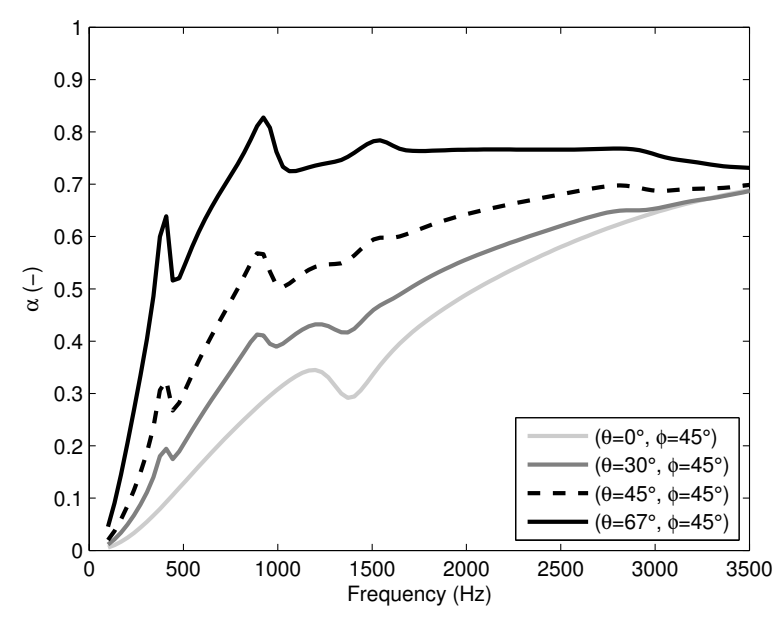

(b)

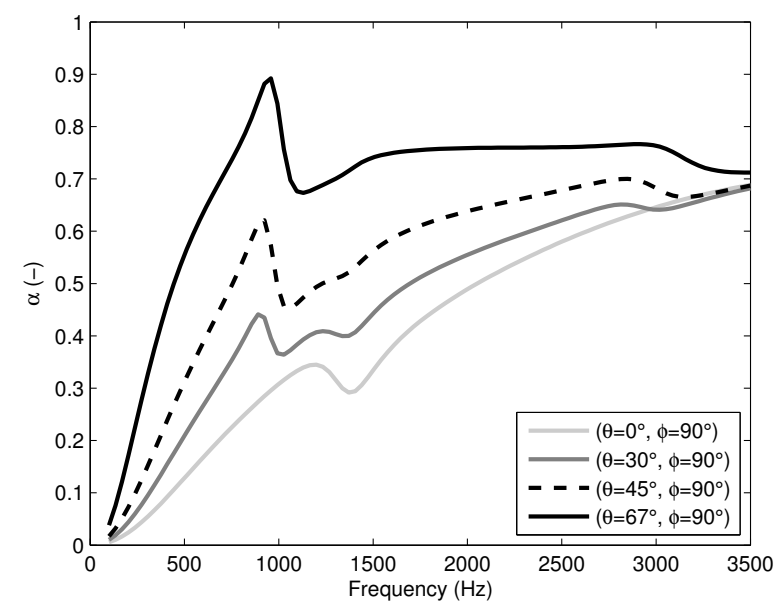

(c)

Figure 5: Numerical results for lamellas network according to the frequency and the inclination angle $\theta \in$ $[0,30,45,67]^{\circ}$ for a) $\phi=0^{\circ}$ and double porosity results at normal incidence $(--)$, b) $\phi=45^{\circ}$ and c) $\phi=90^{\circ}$. 


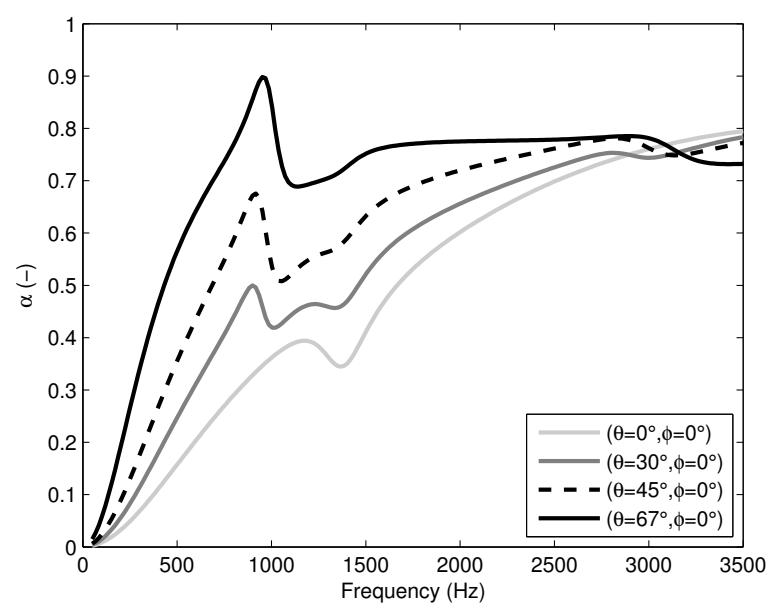

Figure 6: Numerical results for homogeneous porous material according to the frequency and the inclination angle $\theta \in[0 ; 30 ; 45 ; 67]^{\circ}$.

mated by $f_{s}=\frac{1}{2 \pi} \frac{\sqrt{E / 2 \rho_{1}}}{4 h}$, which gives $976 \mathrm{~Hz}$. Finally, Figure 5(c) shows that when $\phi=90^{\circ}$, the bending mode resonance disappears, and only the shear mode peak is present. In that case, the incident waves oriented along the lamellas axis cannot excite the bending mode. Note that these results are close to those given in Figure 6 for a homogeneous layer.

To better analyze the dissipation of mechanisms related to each peak, the absorption coefficient fractions [41] related to the viscous, thermal and structural dissipation are presented in Figure 7. A clear peak of the structural and viscous dissipation fractions can be seen around each mode, the latter being related to the relative velocity between both phases. In contrast, the thermal dissipation is hardly affected by the skeleton motion.

\subsection{Experimental results}

Figure 8 shows the experimental results for the lamella network for three azimuthal angles $\phi=[0,45,90]^{\circ}$ and for the inclination angle $\theta=45^{\circ}$ and $\theta=67^{\circ}$. The estimated sound absorption coefficients are averaged over $1 / 12$ octave bands. Although the sample necessarily includes some manufacturing imperfections, the trends observed are similar to those identified with the numerical results (see Figure 5: dashed lines for $\theta=45^{\circ}$ and continuous black lines for $\theta=67^{\circ}$ ). A peak before 1000 $\mathrm{Hz}$ (related to the shear mode) can be seen except when the incidence is normal to the axis of the lamellas. Conversely, a peak around $500 \mathrm{~Hz}$ can be seen clearly except when the incidence is along the lamellas axis. Using laser vibrometer measurements, this peak was experimentally identified as being linked to the bending mode of the lamellas (as presented in section 3.3).

It can also be noticed that the absorption coefficient can be negative below $500 \mathrm{~Hz}$. Indeed, the reflection co-

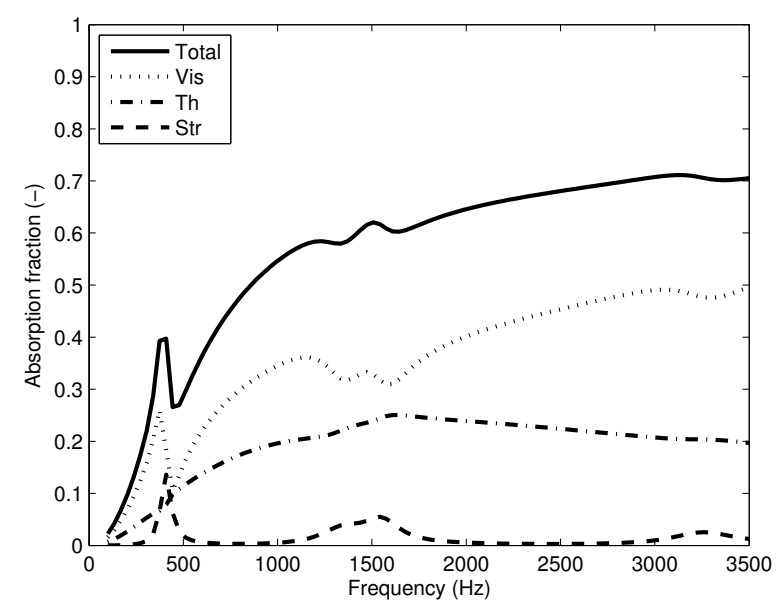

(a)

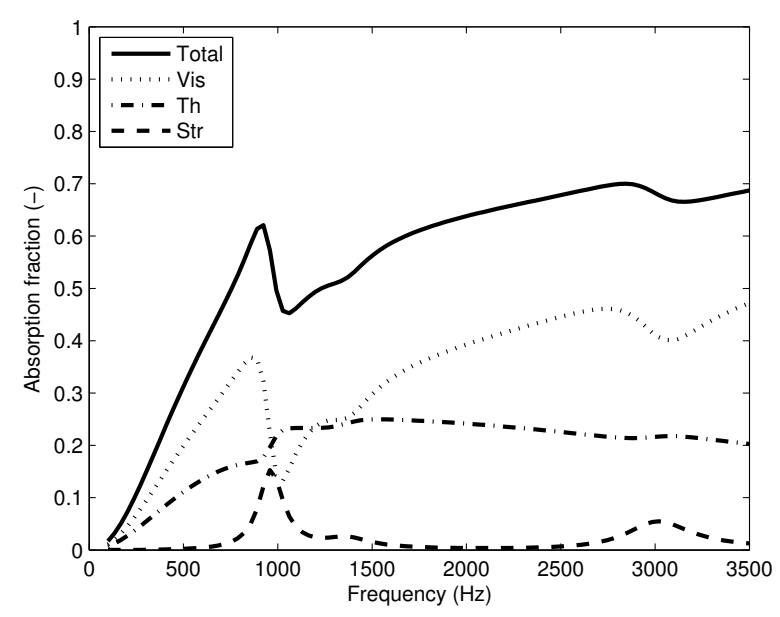

(b)

Figure 7: Absorption fraction for each dissipation mechanism (viscous, thermal and structural) for $\theta=45^{\circ}$ and a) $\phi=0^{\circ}$, b) $\phi=90^{\circ}$.

efficient measured can differ from that provided with the plane-wave hypothesis when the sound source is close to the porous material surface. This can be attributed to the fact that spherical waves do not reflect only specularly when the distance between the sound source and the material surface is small compared to the wavelength [42].

\section{Conclusion}

In this paper, the sound absorption of a poroelastic lamella network was studied. It was shown that this arrangement provides additional sound absorption below the quarter wavelength resonance within the thickness of the sample, compared with a uniform material arrangement.

A sample of $1.2 \mathrm{~m}$ by $1.2 \mathrm{~m}$ made of 65 melamine foam lamellas was tested using a technique based on acoustic field synthesis. This method allows measuring the ab- 


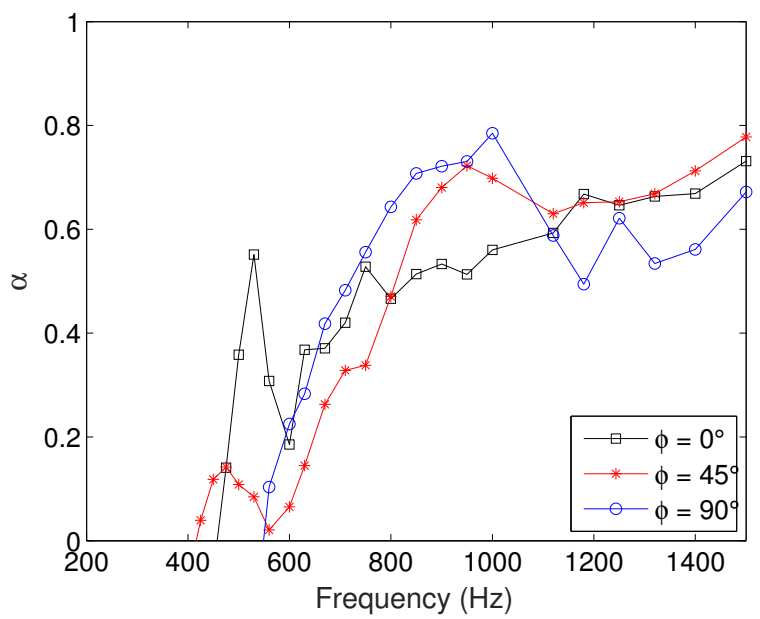

(a)

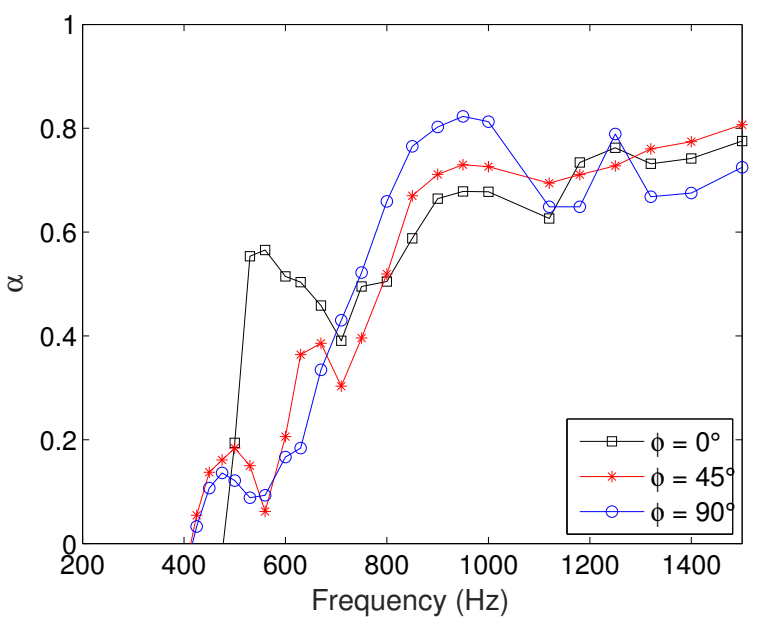

(b)

Figure 8: Measured absorption coefficient for the lamella network according to the frequency and the azimuthal angle $\phi=[0,45,90]^{\circ}$ for an inclination angle a) $\theta=45^{\circ}$, b) $\theta=67^{\circ}$.

sorption coefficient under acoustic plane waves at oblique incidence in free field conditions. The results were in good agreement with the periodic numerical model.

The simulations showed that this additional dissipation is a combination of structural and viscous dissipation within the lamellas. Indeed, at oblique incidence, the lamellas exhibited both a shear and a bending resonance. The frequency of the former was linked to the thickness of the sample, whereas the second was a function of both the width and the thickness of the sample. The two physical dimensions of the lamellas could be used to facilitate the adjustment of additional absorption frequencies appearing below the classical quarter wavelength resonance.

Future works will be devoted to alternative geometries. For instance, rigid splitters [17] can be replaced by air gaps, leading to a horizontal lamella network of different sizes that can be excited at all incidence angles and be tuned at different frequencies to enlarge the frequency range of the additional absorption. The network should also be designed by optimizing its properties involved in double porosity dissipation [39] with air gap size, periodicity and material resistivity or by applying critical coupling [20] to the bending mode to obtain maximum sound absorption.

Finally, the experimental technique used to obtain the absorption coefficient at oblique incidence will be enhanced in the low frequency range by optimizing the post-processing step or using a more precise model of the sound reflection. The capacity to evaluate non specular reflection, which can occur with metamaterials or metasurfaces, will also be tested.

\section{Acknowledgements}

This article is based upon work from COST Action DENORMS - CA15125, supported by COST (European Cooperation in Science and Technology). The authors are grateful for the funds received from the French Research National Agency and the Research Foundation for Aeronautics and Space in the framework of the Metaudible project (ANR-13-BS09-0003-01) and the scientific council of Université de Technologie de Compiègne for supporting the mission at Sherbrooke University.

\section{References}

[1] G. Ma and P. Sheng. Acoustic metamaterials: from local resonances to broad horizons. Sci. Adv., 2:e1501595, 2016.

[2] S. A. Cummer, J. Christensen, and A. Alù. Controlling sound with acoustic metamaterials. Nat. Rev. Mats., 1:16001, 2016.

[3] N. Atalla, R. Panneton, F. C. Sgard, and X. Olny. Acoustic absorption of macro-perforated porous materials. J. Sound Vib., 243(4):659-678, 2001.

[4] X. Olny and C. Boutin. Acoustic wave propagation in double porosity media. J. Acoust. Soc. Am., 114(1):73-89, 2003.

[5] F. C. Sgard, X. Olny, N. Atalla, and F. Castel. On the use of perforations to improve the sound absorption of porous materials. Appl. Acoust., 66:625-651, 2005 .

[6] S. Griffiths, B. Nennig, and S. Job. Porogranular materials composed of elastic Helmholtz resonators for acoustic wave absorption. J. Acoust. Soc. Am., 141(1):254-264, 2017.

[7] B. Nennig, Y. Renou, J.-P. Groby, and Y. Aurégan. A mode matching approach for modeling two dimensional porous grating with infinitely rigid or soft inclusions. J. Acoust. Soc. Am., 131(5):3841$3852,2012$. 
[8] J.-P. Groby, A. Duclos, O. Dazel, L. Boeckx, and L. Kelders. Enhancing absorption coefficient of a backed rigid frame porous layer by embedding circular periodic inclusions. J. Acoust. Soc. Am., 130:3771-3780, 2011.

[9] T. Wu, T. J. Cox, and Y. W. Lam. A profiled structure with improved low frequency absorption. $J$. Acoust. Soc. Am., 110(6):3064-3070, 2001.

[10] P. Leclaire, O. Umnova, T. Dupont, and R. Panneton. Acoustical properties of air-saturated porous material with periodically distributed dead-end pores. J. Acoust. Soc. Am., 137(4):1772-1782, 2015.

[11] J. Yang, J. S. Lee, and Y. Y. Kim. Metaporous layer to overcome the thickness constraint for broadband sound absorption. J. Appl. Phys., 117(17):174903, 2015 .

[12] C. Boutin. Acoustics of porous media with inner resonators. J. Acoust. Soc. Am., 134(6):4717-4729, 2013.

[13] C. Boutin and F.-X. Becot. Theory and experiments on poro-acoustics with inner resonators. Wave Motion, 54(0):76-99, 2015.

[14] J.-P. Groby, B. Nennig, C. Lagarrigue, B. Brouard, O. Dazel, and V. Tournat. Enhancing the absorption properties of acoustic porous plates by periodically embedding Helmholtz resonators. J. Acoust. Soc. Am., 137(6):273-280, 2015.

[15] O. Doutres, N. Atalla, and H. Osman. Transfer matrix modeling and experimental validation of cellular porous material with resonant inclusions. J. Acoust. Soc. Am., 137(6):3502-3513, 2015.

[16] J.-P. Groby, W. Huang, A. Lardeau, and Y. Aurégan. The use of slow waves to design simple sound absorbing materials. J. Appl. Phys., 117(12):124903, 2015.

[17] J. Yang, J. S. Lee, and Y. Y. Kim. Multiple slow waves in metaporous layers for broadband sound absorption. J. Phys. D: Appl. Phys., 50(1):015301, 2017.

[18] N. Jiménez, W. Huang, V. Romero-García, V. Pagneux, and J.-P. Groby. Ultra-thin metamaterial for perfect and quasi-omnidirectional sound absorption. Appl. Phys. Lett., 109(12):121902, 2016.

[19] J. Mei, G. G. Ma, M. Yang, Z. Yang, W. Wen, and P. Sheng. Dark acoustic metamaterials as super absorbers for low-frequency sound. Nature commun., 3:756, 2012.

[20] V. Romero-García, G. Theocharis, O. Richoux, A. Merkel, V. Tournat, and V. Pagneux. Perfect and broadband acoustic absorption by critically coupled sub-wavelength resonators. Sci. Rep., 6(5):19519, 2016.
[21] T. Weisser, J.-P. Groby, O. Dazel, F. Gautier, E. Deckers, S. Futatsugi, and L. Monteiro. Acoustic behavior of a rigidly backed poroelastic layer with periodic resonant inclusions by a multiple scattering approach. J. Acoust. Soc. Am., 139(2):617-629, 2016.

[22] J. Christensen, V. Romero-García, R. Picó, A. Cebrecos, F. J. García de Abajo, N. A. Mortensen, M. Willatzen, and V. J. Sánchez-Morcillo. Extraordinary absorption of sound in porous lamellacrystals. Sci. Rep., 4:4674, 2014.

[23] N. Dauchez and B. Nennig. Absorption of a poroelastic material with lateral air gaps. In INTERNOISE and NOISE-CON Congress and Conference Proceedings, pages 4762-4768. Institute of Noise Control Engineering, 2012.

[24] J.-F. Allard and N. Atalla. Propagation of Sound in Porous Media: Modeling Sound Absorbing Materials (second edition), page 372pp. John Wiley \& Sons, Chichester, 2009

[25] J.-P. Berenger. A perfectly matched layer for the absorption of electromagnetic waves. J. Comput. Phys., 114(2):185 - 200, 1994.

[26] N. Atalla, M. A. Hamdi, and R. Panneton. Enhanced weak integral formulation for the mixed (u, p) poroelastic equations. J. Acoust. Soc. Am., 109(6):3065-3068, 2001.

[27] A. C. Hennion, R. Bossut, J. N. Decarpigny, and C. Audoly. Analysis of the scattering of a plane acoustic wave by a periodic elastic structure using the finite element method : application to compliant tube gratings. J. Acoust. Soc. Am., 87(5):1861-1870, 1990 .

[28] P. R. Heyl, V. L. Chrisler, and W. F. Snyder. The absorption of sound at oblique angles of incidence. J. Res. Natl. Bur. Stand., 4 (RP149):289-295, 1929.

[29] T. Schultz, L. N. Cattafesta, and M. Sheolak. Modal decomposition method for acoustic impedance testing in square ducts. J. Acoust. Soc. Am., 120:3750$3758,2006$.

[30] J. Prisutova, K. Horoshenkov, J.-P. Groby, and B. Brouard. A method to determine the acoustic reflection and absorption coefficients of porous media by using modal dispersion in a waveguide. $J$. Acoust. Soc. Am., 136:2947-2958, 2014.

[31] E. Brandão, A. Lenzi, and S. Paul. A review of the in situ impedance and sound absorption measurement techniques. Acta Acust. United Ac., 101:443-463, 2015.

[32] J.-F. Allard and Y. Champoux. In-situ two microphone technique for the measurement of the acoustic 
surface impedance of materials. Noise Control Eng. J., 32(1):15-23, 1989.

[33] A. Richard, E. Fernandez-Grande, J. Brunskog, and C.-H. Jeong. Estimation of surface impedance at oblique incidence based on sparse array processing. J. Acoust. Soc. Am., 141(6):4115-4125, 2017.

[34] M. Tamura. Spatial fourier transform method of measuring reflection coefficients at oblique incidence. i. theory and numerical examples. J. Acoust. Soc. Am., 88:2259-2264, 1990.

[35] O. Robin, A. Berry, O. Doutres, and N. Atalla. Measurement of the absorption coefficient of sound absorbing materials under a synthesized diffuse acoustic field. J. Acoust. Soc. Am., 136(1):EL13-EL19, 2014.

[36] A. Berry, R. Dia, and O. Robin. A wave field synthesis approach to reproduction of spatially correlated sound fields. J. Acoust. Soc. Am., 131(2):1226-1239, 2012 .

[37] O. Robin, A. Berry, and S. Moreau. Reproduction of random pressure fields based on planar nearfield acoustic holography. J. Acoust. Soc. Am., 133(6):3885-3899, 2013.

[38] P. A. Nelson and S. H. Yoon. Estimation of acoustic source strength by inverse methods: Part i, conditioning of the inverse problem. J. Sound Vib., 233(4):639-664, 2000.

[39] B. Nennig, R. Binois, E. Perrey-Debain, and N. Dauchez. A homogenization method used to predict the performance of silencers containing parallel splitters. J. Acoust. Soc. Am., 137(6):3221-3231, 2015 .

[40] M. Geradin and D.J. Rixen. Mechanical Vibrations: Theory and Application to Structural Dynamics. Wiley, 2015.

[41] O. Dazel, F. Sgard, F.-X. Becot, and N. Atalla. Expressions of dissipated powers and stored energies in poroelastic media modeled by $(\mathrm{u}, \mathrm{u})$ and $(\mathrm{u}, \mathrm{p})$ formulations. J. Acoust. Soc. Am., 123(4):2054-2063, 2008.

[42] R. Dragonetti, R. Opdam, M. Napolitano, R. Romano, and M. Vorländer. Effects of the wave front on the acoustic reflection coefficient. Acta Acust. United Ac., 102:675-687, 2016. 